Musées, Patrimoine et Culture scientifiques et techniques

177 | 2018

mai-juin 2018

\title{
Le Fab Lab, l'agitateur du centre de sciences
}

\section{Bruno Dosseur}

URL : http://journals.openedition.org/ocim/2533

DOI : 10.4000/ocim.2533

ISSN : 2108-646X

\section{Éditeur}

OCIM

Édition imprimée

Date de publication : 1 mai 2018

Pagination : 13-17

ISSN : 0994-1908

Référence électronique

Bruno Dosseur, « Le Fab Lab, l'agitateur du centre de sciences », La Lettre de I'OCIM [En ligne],

177 | 2018, mis en ligne le 01 mai 2019, consulté le 05 juin 2019. URL : http://journals.openedition.org/ ocim/2533; DOI : 10.4000/ocim.2533

Ce document a été généré automatiquement le 5 juin 2019

Tous droits réservés 


\title{
Le Fab Lab, l'agitateur du centre de sciences
}

\author{
Bruno Dosseur
}

Relais d'sciences a organisé des évènements sur l'agglomération caennaise pour mobiliser les communautés de makers et faire connaître le Fab Lab à un large public comme ici, en octobre 2012, lors du festival "Nördik Numérik".

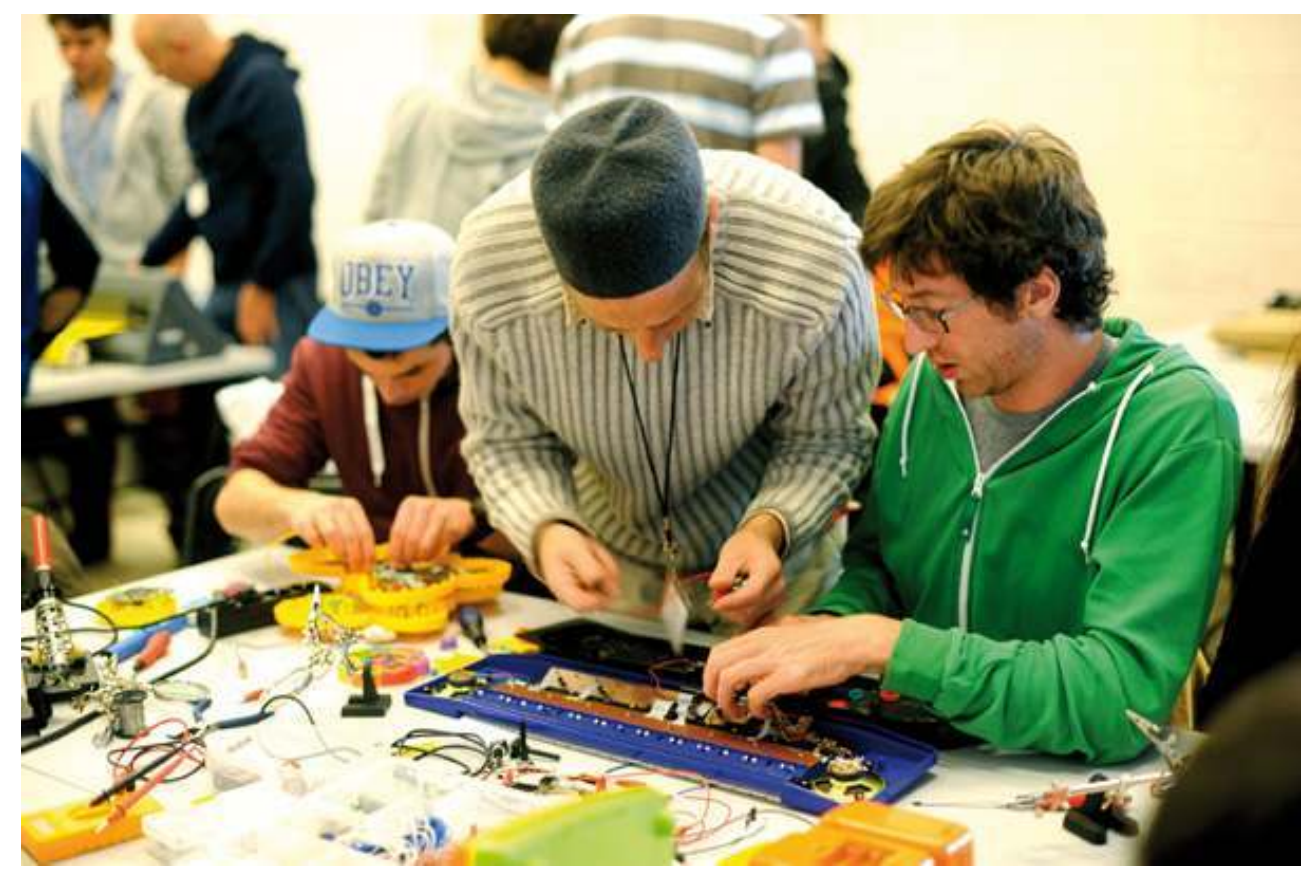

(c) Relais d'sciences/F. Castel.

\section{Préambule}

1 En 2011, au moment de l'écriture du projet Inmédiats ${ }^{1}$ les Fab Labs sont encore une curiosité naissante. La Casemate, à Grenoble, est alors précurseur en la matière en étant 
le premier CCSTI à proposer un Fab Lab ouvert aux publics. Inmédiats s'est donc appuyé sur cette initiative pour faire le pari de mettre le développement des Fab Labs au cœur de son projet de renouvellement de la médiation culturelle en sciences et techniques à l'attention des publics, tout particulièrement des jeunes adultes. Quelques années après, le concept de Fab Lab n'est plus une nouveauté et la France s'illustre par un développement particulièrement important de ces ateliers ouverts à tous conceptualisés par le Massachusetts Institute of Technology (MIT). Les Fab Labs Inmédiats ont grandi et se sont insérés dans la vie quotidienne de plusieurs centres de culture scientifique et technique. Le propos de cet article n'est pas de décréter des généralités sur les Fab Labs, mais de s'intéresser à un cas particulier, celui que nous connaissons au sein du Dôme ${ }^{2}$, centre de culture scientifique de nouvelle génération né de ce programme Inmédiats en 2015.

\section{Genèse}

Le choix d'intégrer un Fab Lab à la construction de ce nouveau bâtiment s'est fait sur une double conviction : le sentiment que le format "atelier" était un mode de médiation culturelle trop peu exploité dans la relation aux publics et l'intuition que le Fab Lab pouvait permettre la rencontre avec des publics et partenaires complètement nouveaux pour un CCSTI. Malgré l'expérience naissante de nos collègues de La Casemate à Grenoble, le sujet était si nouveau qu'il nous a semblé indispensable de pré-configurer un espace de $30 \mathrm{~m} 2$ pendant 18 mois avant d'intégrer nos nouveaux espaces au Dôme.

Le Dôme est le premier centre de sciences de nouvelle génération. Il développe $2100 \mathrm{~m} 2$ sur 4 niveaux, dont 1500 dédiés aux activités avec les publics.

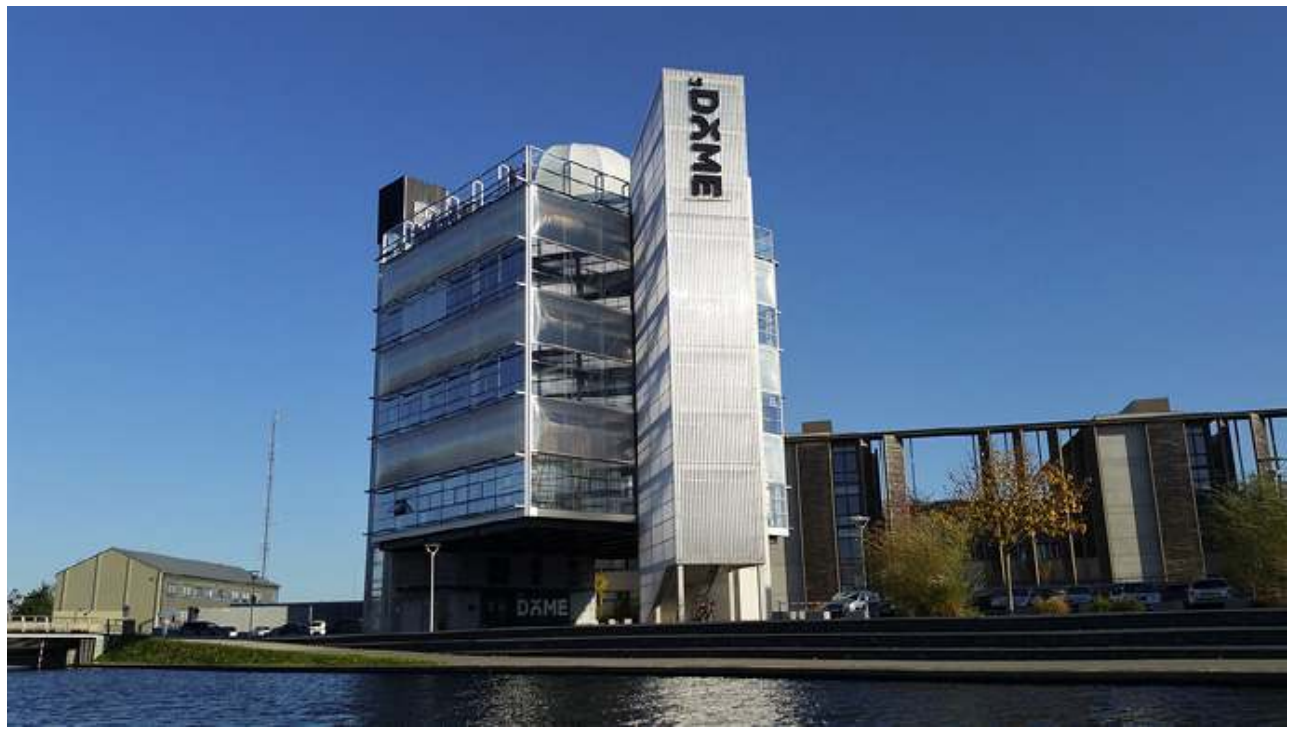

(C) Relais d'sciences/G. Dupuy

Tout était effectivement à inventer pour une équipe de CCSTI : quelles machines, pour quels publics et usages, quelle relation au réseau des Fab Labs, comment monter l'équipe en compétences, quel modèle économique et tarification des services, quel type d'animation et quelles propositions aux publics, quel outil de gestion ou quels horaires d'ouverture ? Cette préfiguration du Fab Lab s'est effectuée au cœur du Forum digital, une 
Cantine numérique avec un espace de co-working animé par la Communauté urbaine Caen la mer au sein d'un campus technologique. Autrement dit un lieu professionnel non prédestiné à l'accueil du grand public.

\section{Perturbateur systémique}

4 Le Dôme a été conçu par Relais d'sciences et l'agence d'architecture Bruther et se développe avec le soutien de la Région Normandie. Il est pensé comme un lieu modulaire permettant de développer essentiellement des activités en science et technique sous forme d'ateliers avec les publics particuliers et professionnels. La présence d'un atelier de fabrication était donc tout à fait pertinente au regard de ce projet. Le Fab Lab occupe environ $150 \mathrm{~m} 2$ des $2100 \mathrm{~m} 2$ du Dôme mais peut "déborder" sur l'intégralité de l'étage de $400 \mathrm{~m} 2$ où il se situe et ses $100 \mathrm{~m} 2$ de salles de réunion. Il s'inscrit ainsi dans la dynamique de modularité prévue pour l'ensemble du bâtiment. Sa présence est, pour les publics et partenaires, la partie visible de l'iceberg Dôme et constitue ainsi un élément perturbateur intéressant à plusieurs titres. Le Fab Lab est d'abord un agitateur organisationnel objectif. En effet, le rythme de fonctionnement d'un Fab Lab ouvert aux publics impose une régularité d'accessibilité. À côté de l'activité essentiellement évènementielle du reste du Dôme, le Fab Lab impose les horaires habituels d'ouverture pour les publics (les aprèsmidis du mercredi au samedi). Il fixe également une partie conséquente de la masse salariale indispensable à sa maintenance, son fonctionnement en toute sécurité et à l'accompagnement des usagers. Sur le plan architectural, la présence d'un Fab Lab est loin d'être anodine. Il faut en effet penser la circulation des publics, l'évacuation des fumées et déchets, la cohabitation bruyante des machines avec les autres espaces... La simple présence de l'atelier et de ses machines fixe les espaces laissés modulaires dans les autres étages du Dôme. Le fab Lab influe donc très fortement sur la conception et la scénographie des lieux et leur évolutivité. 
Les salariés du Dôme affectés au Fab Lab assurent l'accueil et la médiation pour favoriser la participation de nouveaux publics.

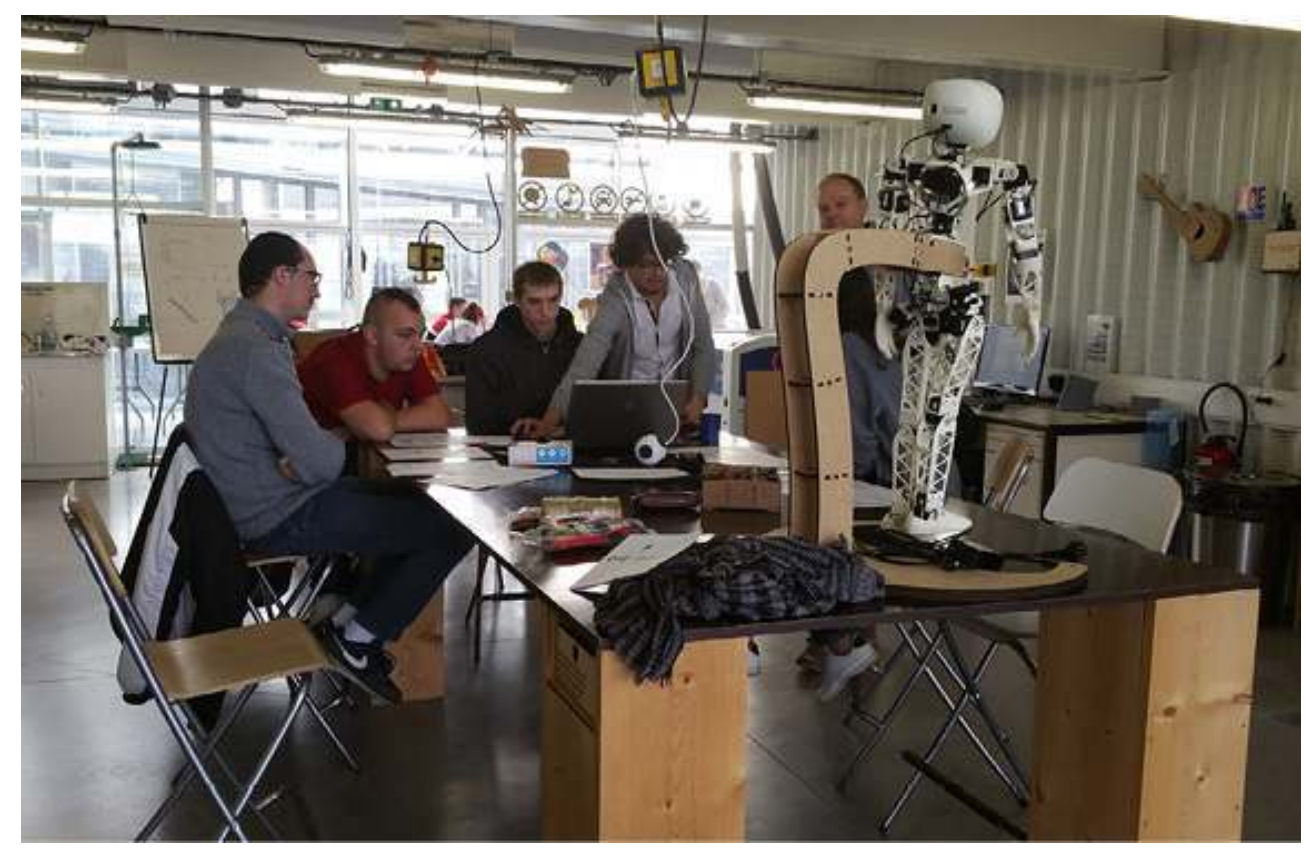

(C) Relais d'sciences/G. Dupuy

Il est également un agitateur économique. Par sa fonction, le Fab Lab permet une ouverture plus aisée du centre de sciences vers le monde industriel, mais également celui de la formation professionnelle, de l'insertion ou du handicap. Il favorise ainsi la naissance de nouveaux partenariats comme c'est le cas, par exemple, avec le Pôle ATEN (Pôle Innovation \& Numérique de la Chambre de métiers) ou la déclinaison de nouvelles prestations de service pour l'entreprise. Dans le cas du Dôme, il a participé à repenser le modèle économique global du centre de science en raison de son positionnement sur des prestations entrant largement dans le champ concurrentiel. Il reste toutefois un service déficitaire du fait de l'importance de la masse salariale nécessaire au regard de prestations volontairement proposées à des prix accessibles pour favoriser l'usage par le plus grand nombre. L'équilibre global est assuré par d'autres services du Dôme. Ce choix économique stratégique lui confère le rôle essentiel d'être une porte d'entrée visible vers les autres activités du centre de sciences. Cette invitation aux nouveaux publics a nécessité le renforcement rapide de l'équipe grâce à l'aide de trois services civiques qui expérimentent de nouvelles formes d'accueil et de fidélisation du public. 
Le Dôme organise régulièrement des ateliers à destination de publics non familiers des ateliers de fabrication numérique comme ici avec des lycéennes en filière "Métiers de la mode" lors d'un workshop consacré aux textiles connectés avec le collectif DataPaulette.

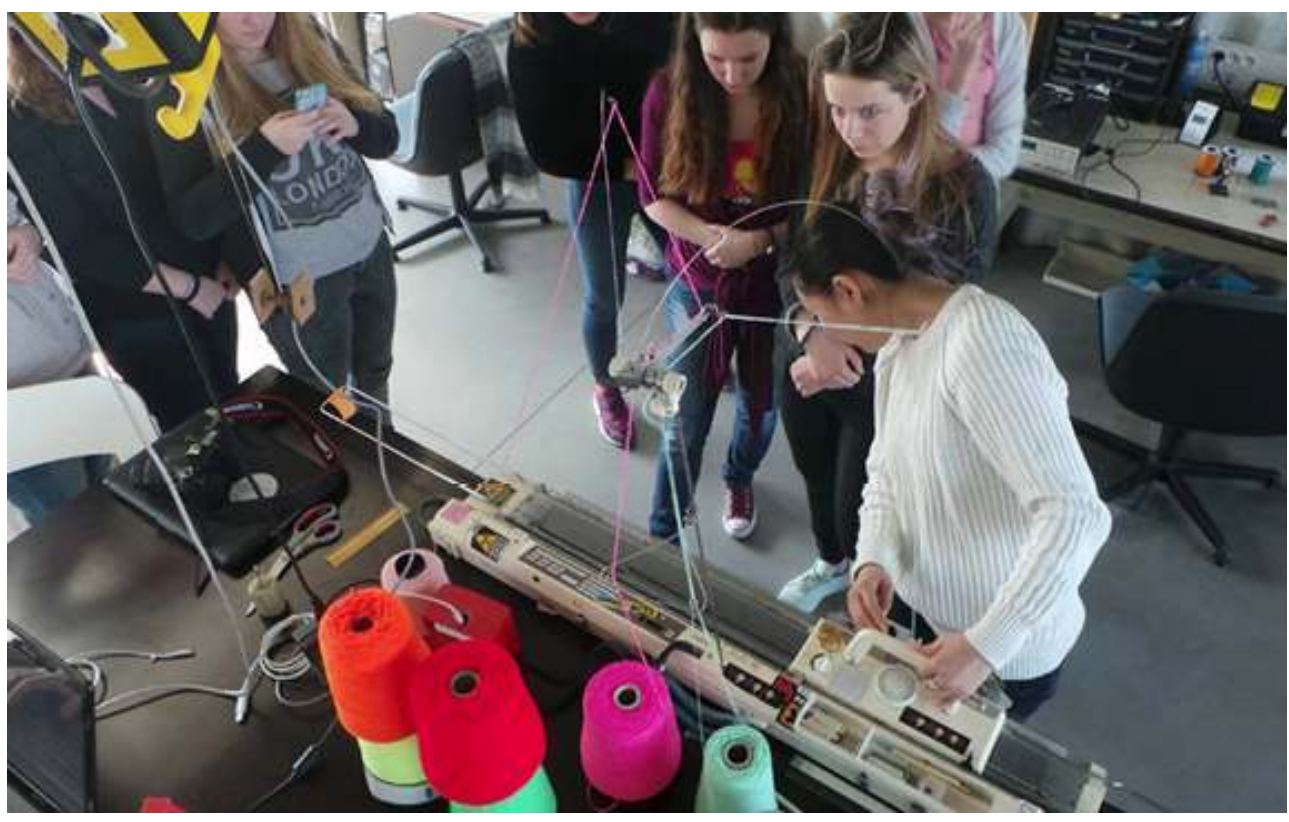

(C) Relais d'sciences/G. Dupuy

$6 \quad$ Il est enfin et surtout un agitateur symbolique et culturel. Le Fab Lab envoie plusieurs messages intrinsèques aux publics. Il est le lieu de la mise en pratique, du Do it Yourself et, dans le meilleur des cas, du Do it with others. Il est aussi le lieu originel des makers, de la technicité, parfois de la démarche underground ou empirique. Sa population naturelle est plutôt masculine, jeune et technophile. L'entrée et l'acceptation de nouveaux publics dans cet espace est donc un enjeu premier de médiation. La constitution de communautés d'échanges relève rapidement du challenge et nécessite une dynamique d'animation volontariste (workshops, atelier ouvert, découvertes de groupes, intégration à des festivals et opérations évènementielles). Lorsque l'on est étranger à ce type de lieu, la primo-arrivée est un moment charnière qui peut séduire ou dissuader. On constate par ailleurs qu'une première rencontre très positive avec le lieu peut être suivie de plusieurs mois avant d'induire une participation active sur un projet, même individuel. Le Fab Lab peut donc susciter un mouvement de rejet ou de désintérêt tout autant que d'enthousiasme. Dans les deux cas, la transformation en participation récurrente restera un enjeu permanent. Il existe donc un acte de médiation indispensable pour favoriser la participation des publics au sein des espaces Fab Lab, depuis la primo-arrivée (qui doit être transformée) jusqu'à la participation active, sous forme de partage de compétences, de documentation de projets ou d'implication dans un projet tiers par exemple. En bref, les choses n'arrivent pas complètement par hasard et la sérendipité ne se décrète pas. La médiation culturelle est certainement la contribution majeure que peuvent proposer les centres de culture scientifique à ce sujet. 


\section{Perturbateur de la représentation d'un centre de culture scientifique}

7 L'entrée des Fab Labs dans le monde de la CSTI n'est cependant pas complètement une nouveauté. Plus exactement, la forme de médiation proposée autour du Faire, individuellement ou par petits groupes, avec une récurrence de participation et des médiateurs / accompagnateurs techniques existe depuis longtemps. On pense bien sûr aux acteurs de l'éducation populaire présents dans le champ de la CSTI, au premier rang desquels Planète Sciences. Toutefois, le positionnement des Fab Labs amène les CCSTI à dépasser le cadre habituel de leurs partenariats. Il ouvre symboliquement une relation nouvelle avec les acteurs de l'innovation et de la formation. En cela, il perturbe la représentation que l'on a d'un centre de culture scientifique. L'adressage du public n'est plus seulement celui des familles, des enseignants et des passionnés de sciences et techniques mais s'étend aux acteurs industriels, aux artisans, aux start-up, aux organismes de formation et d'insertion...

Le Dôme a mis en place un programme de formations techniques avec un service de la Chambre départementale des métiers et de l'artisanat (Pôle ATEN) pour accompagner les professionnels dans la prise en main des nouvelles technologies.

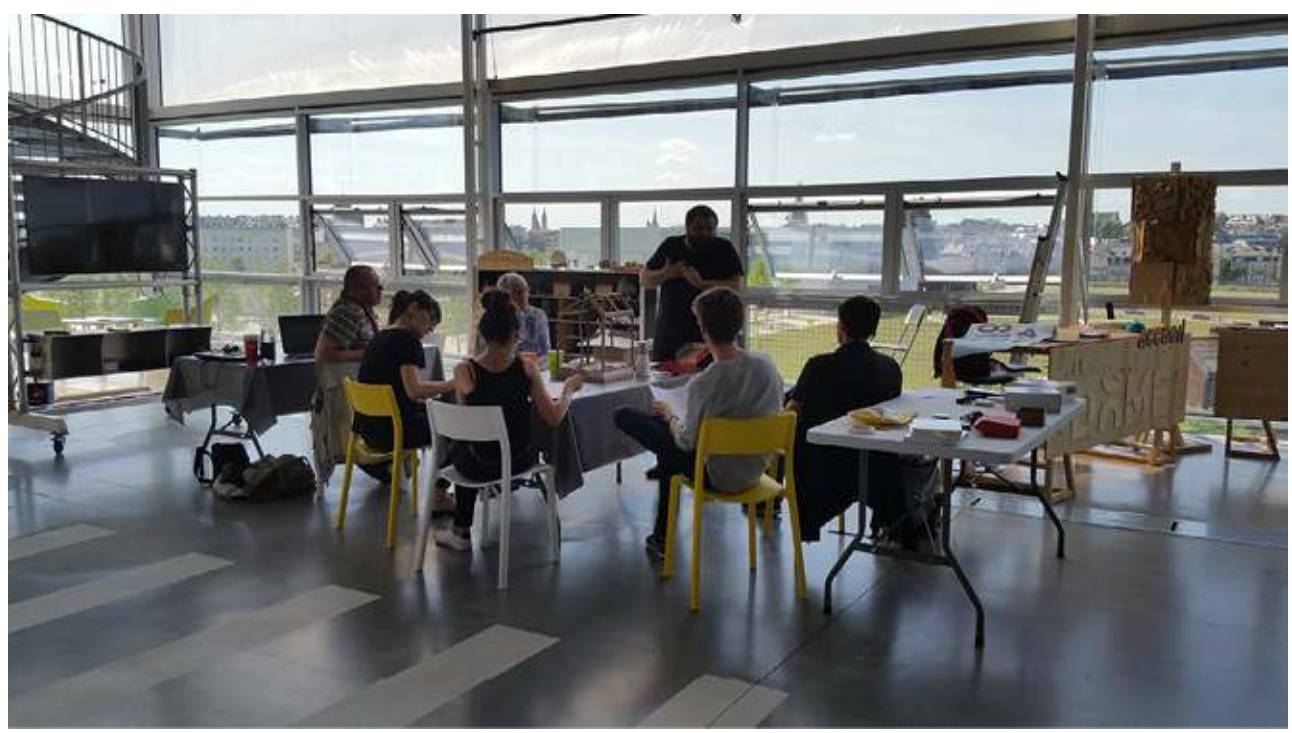

(c) Relais d'sciences/G. Dupuy 


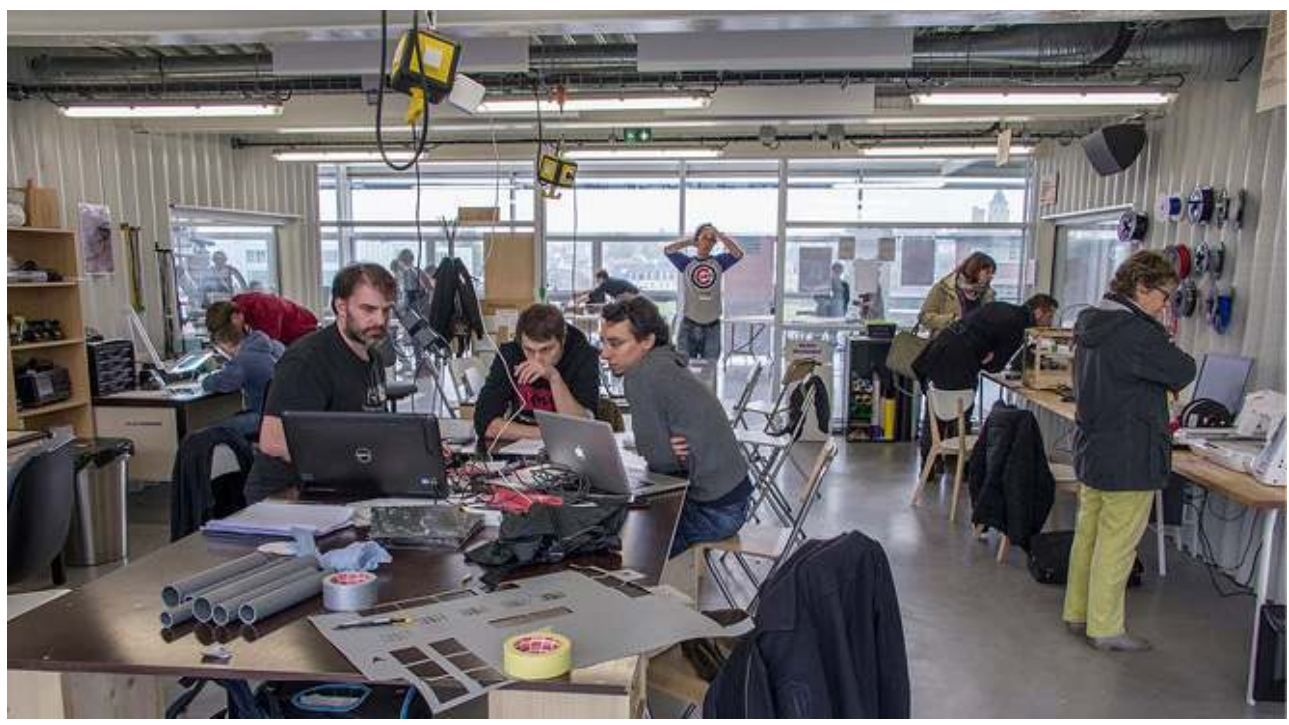

(c) V. Connetable

8 Ce déplacement symbolique impacte la communication d'un centre comme Le Dôme. La place du Fab Lab doit être définie pour permettre la rencontre constructive des différents publics comme les makers, les publics traditionnels de la CSTI, les entrepreneurs, chercheurs et artistes. De plus, l'existence d'un espace physique d'atelier, ouvert régulièrement, tend à prendre une place plus importante en matière de visibilité que le flux public qu'il génère, par essence plutôt faible au regard des autres activités. Deux stratégies peuvent alors s'affronter : faut-il souligner l'existence d'un Fab Lab pour donner un signal fort au réseau $\mathrm{Fab} \mathrm{Lab}$ ou, au contraire, le fondre à une entité plus large, tiers-lieu de culture scientifique et d'innovation ouvert au public ? La dénomination même de Fab Lab et sa programmation propre peuvent devenir un questionnement. Il n'est ainsi pas rare que des partenaires ou des publics restreignent leur vision du Dôme à celle d'un Fab Lab alors même qu'en interne les animateurs du Fab Lab peuvent avoir le sentiment de ne pas être assez visibles. Le sujet va très loin puisque la plateforme même de pilotage comme Fab Manager est construite pour faire du Fab Lab une entité propre et non un service d'un lieu qui propose une programmation plus large. Si l'on comprend bien que le Fab Lab bouscule les habitudes d'un centre de culture scientifique, en quoi estil une nouvelle proposition dans la relation au monde de la recherche et de l'innovation?

\section{Perturbateur de la relation à la recherche et à l'innovation}

Plusieurs choix ont été faits au Dôme dès la conception du Fab Lab : la mixité des publics particuliers et professionnels, le conventionnement autour de la venue régulière de publics 15-25 ans (publics fragilisés, étudiants, apprentis), la discrimination tarifaire selon les typologies de publics, le choix de l'open source, la mobilisation et l'animation de l'ensemble des Fab Lab de Normandie au sein d'un réseau, les partenariats de formation/ initiation avec les structures professionnelles, la mobilisation lors des phases de prototypage de démarche Living Lab, la définition de services spécifiques à destination des professionnels. Tous ces éléments sont de nature à renforcer les capacités du Dôme à proposer l'accès aux machines du Fab Lab dans une démarche d'innovation ouverte à 
l'ensemble des parties prenantes du territoire, des chercheurs aux industriels en passant par les collectivités et les communautés créatives.

La création d'un Fab Lab a permis au Dôme de se rapprocher des acteurs de la formation professionnelle. Un partenariat existe par exemple depuis plusieurs années avec le Pôle formation de l'Union des Industries et Métiers de la Métallurgie de Normandie.

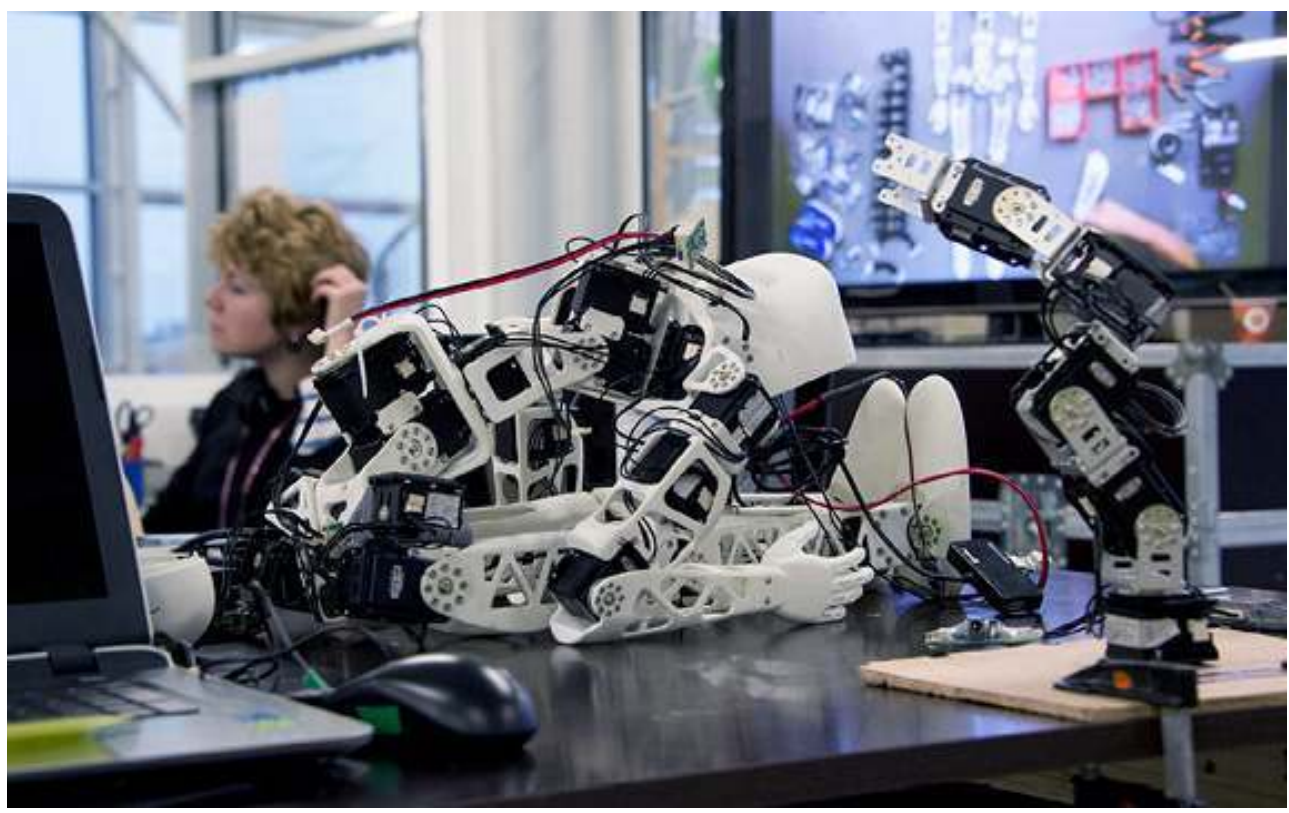

(c) V. Connetable

10 La proposition de médiation tend à inviter ces parties prenantes à co-construire avec les publics autour de projets concrets de recherche ou d'innovation technologique. Les publics deviennent ainsi les participants à un projet ouvert, dont le résultat sera partagé en Licences Creatives Commons. L'existence d'un espace de prototypage membre d'un réseau international et inclus dans un lieu d'innovation ouvert aux publics comme le Dôme offre de nombreuses possibilités nouvelles : accompagnement de l'entrepreneuriat étudiant, immersion dans une ambiance Team building de salariés d'entreprises, ouverture à de nouveaux sujets technologiques ou scientifiques... Mais l'intérêt majeur réside dans la capacité à faire de la culture scientifique et technique avec des sujets en prise réelle avec les activités du territoire. Il n'est plus nécessaire de conceptualiser un sujet pour le proposer sous une forme didactique. Le passage à la réalisation de prototype facilite une représentation immédiate et concrète du sujet scientifique ou technique abordé. Le processus essai-erreur de la démarche expérimentale fait partie de la démarche et peut participer à la résolution de problèmes très concrets. Une forme, parmi d'autres, de contribution à la modification du réel. Ainsi, au Fab Lab du Dôme, le dispositif FFTBox $^{3}$ permet de capter un son et de le transformer en signal lumineux. Il a été utilisé à l'occasion de plusieurs concerts, notamment avec Le Cargö, Scène de musiques actuelles de Caen. Le projet Hope \& Bike ${ }^{4}$, soutenu par la Fondation Orange Solidarité et lauréat du prix EDF Pulse, développe un kit d'électrification et de gestion de l'énergie pour vélos adaptable pour d'autres objets roulants. 


\section{Perturbateur de la relation aux publics}

11 Ce nouveau positionnement ouvre le champ d'un nouveau rapport aux usagers. Si la constitution de communautés et leur animation reste un sujet délicat, on constate déjà une autre forme d'appropriation du centre de culture scientifique par les publics grâce à la présence du Fab Lab. L'invitation aux publics à passer d'une posture de participant, souvent individuel, à celle de contributeur voire d'initiateur de projet collectif devient un nouvel enjeu de médiation. Il s'agit, en effet, de dépasser la réalisation d'un objet reproduit dans tous les Fab Lab pour amener les usagers à s'emparer des autres dimensions du lieu. Le médiateur présent dans le Fab Lab a donc pour ligne d'horizon un accompagnement à l'implication dans des projets collectifs, une incitation à la découverte d'autres univers techniques et scientifiques, un appui à la documentation et au partage de connaissances acquises au Fab Lab. Cet accompagnement s'avère très individualisé au sein des Fab Labs dans lesquels chaque personne vient avec une intention spécifique. Il nécessite donc des professionnels aguerris à l'accueil, l'animation et l'accompagnement de projet tout autant qu'au fonctionnement technique des machines ou de l'électronique.

\section{Pour conclure}

L'intégration d'un Fab Lab dans un centre de culture scientifique reste un pari. Il faudra encore quelques années, tout particulièrement au Dôme, pour mesurer son impact dans l'acte de médiation en culture scientifique et technique. La démarche même du Fab Lab n'est pas ici en cause. Ce qui interroge, et donc suscite l'intérêt car il peut être le terrain d'une réelle innovation, c'est la position singulière que cet outil occupe dans le champ de la médiation culturelle. Il peut en effet devenir un atelier de production pré-industrielle pour professionnels proposant également des animations autour des nouveaux outils techniques à un public large. Ou alors, il peut devenir un nouveau cœur pour les centres de sciences, lieu de croisement des savoirs et des technologies avec des participants venus de communautés différentes et complémentaires, porteur de projets ancrés sur le territoire et nourris par le champ de la recherche et de l'expérimentation. En somme, un espace créateur de valeur par la création et le partage de connaissances, un activateur de lien social et de projets collaboratifs à l'échelle du territoire, un outil d'accompagnement $\mathrm{du}$ développement économique appropriable par tous. L'expérience ne fait que commencer.

\section{NOTES}

1. Inmédiats est une réalisation commune 2012-2016 de six centres de culture scientifique français (Bordeaux, Grenoble, Caen, Toulouse, Paris et Rennes) dans le cadre du Programme des Investissements d'Avenir. Il a notamment donné naissance au Dôme à Caen et à la plateforme FabManager à Grenoble (www.inmediats.fr). 
2. Le Dôme est un bâtiment réalisé par Relais d'sciences, centre de culture scientifique et technique de Normandie basé à Caen.

3. Voir : http://www.echosciences-normandie.fr/communautes/le-dome/articles/fftbox-sons-etlumieres

4. Voir : http://www.ledome.info/index.php?page=fiche_actualite\&id_manifestation=1976

\section{RÉSUMÉS}

Cette contribution montre que le Fab Lab, lieu de création et de partage des connaissances et pratiques technologiques au sein du centre de sciences de Caen est également un perturbateur. Il modifie les habitudes et les relations qu'entretient l'établissement avec ses publics et ses partenaires de la recherche et de l'innovation.

INDEX

Mots-clés : Fab Lab, réseau, CSTI

\section{AUTEUR}

BRUNO DOSSEUR

Directeur du Dôme à Caen

mbdosseur@ledome.info 